\title{
Network Pharmacology Revealed the mechanism of Platycodon grandiflorum in Lung Cancer Treatment
}

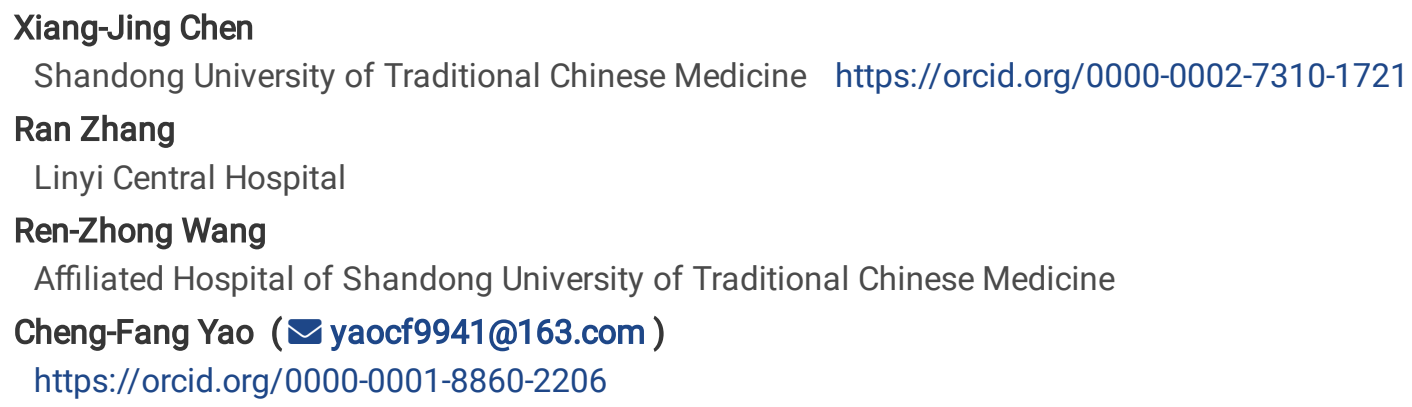

\section{Research}

Keywords: Lung cancer, Traditional Chinese Medicine, Platycodon grandiflorum, network pharmacology theory

Posted Date: August 18th, 2020

DOI: https://doi.org/10.21203/rs.3.rs-58086/v1

License: () (i) This work is licensed under a Creative Commons Attribution 4.0 International License. Read Full License 


\section{Abstract}

Background: Traditional Chinese Medicine has demonstrated increasingly unique advantages in the treatment of lung cancer. Through literature review, it was found out that Platycodon grandiflorum had immunomodulatory and anti-inflammatory effects, and it had a special targeting effect on the lung.

Purpose邓In order to determine the molecular mechanism of Platycodon grandiflorum in lung cancer treatment. Network pharmacology theory was used to do a systematic study.

Methods: The active compounds of Platycodon grandiflorum were screened from TCMSP database. Then, compounds targets were predicted with the assistance of Swiss Target Prediction and STITCH. The targets of lung cancer were screened form TTD and DisGeNET database. The common targets of compounds and lung cancer were screened out for following analysis. A ProteinProtein Interaction (PPI) Network was constructed by STRING. Subsequent to topological analysis, the hub targets were screened out for KEGG pathway and GO Enrichment. Molecular docking by AutoVina was performed to investigate the binding ability between the hub targets and compounds.

Results: There were 4 active compounds screened out, including Acacetin, Spinasterol, cis-Dihydroquercetin and Luteolin. There were 80 targets screened as the common target of compounds and lung cancer. After topological analysis TP53, AKT1, VEGFA, CASP3, IL6, EGFR and MAPK1 were identified as hub targets.

The 7 hub targets might be involved in 81 biological annotation and in the regulation of 23 pathways to intervene lung cancer. The main functional annotation was negative regulation of apoptotic process. Almost all of the pathways were directly or indirectly associated with the PI3K-Akt signal pathway and MAPK signal pathway. According to Affinity score of molecular docking the best binding protein for Luteolin was EGFR, and the best binding protein for Acacetin was CASP3. This meant that the Platycodon grandiflorum was easier to combine these two targets than other targets.

Conclusion: Our study affirmed the effectiveness of Platycodon grandiflorum in treatment of lung cancer from molecular level. And we found that EGFR and CASP3 were the most likely targets for the direct action of Platycodon grandiflorum. The most important pathways that Platycodon grandiflori might interfere with were PI3K-Akt signaling pathway and MAPK signaling pathway.

\section{Introduction}

According to global cancer statistics in 2018 , lung cancer was the most common cancer and the leading cause of cancer death in males, followed by breast and colorectal cancer in females. When both genders were considered altogether, lung cancer still ranked the first ${ }^{[1]}$. Recently, the clinical interventions against lung cancer include surgery, chemotherapy, radiation therapy immunotherapy and target therapy ${ }^{[2,3]}$. Due to the limited effect of these current treatment methods, lung cancer still leads to high mortality and low survival rate. In the meantime, the potential of drug resistance and side effects cannot be ignored. Therefore, the needs for alternative therapeutic approaches have become more and more pressing ${ }^{[4]}$.

Traditional Chinese Medicine (TCM) is a type of complementary and alternative medicine, which is widely used in the treatment of various forms of cancer and widely recognized for its effect. It has been proved that the adjuvant treatment of TCM is effective in improving the overall survival rate among lung cancer patients ${ }^{[5]}$. TCM is capable to enhance host immune response, reduce the adverse reactions of chemotherapy ${ }^{[6]}$, and improve the quality of life for patients ${ }^{[7]}$. A study to investigated the frequencies and patterns of TCM herbal treatment for lung cancer patients in Taiwan found that Platycodon grandiflorum and Mai-Men-Dong-Tang (MMDT) were important TCM herbs by network analysis ${ }^{[8]}$, which suggests that, Platycodon grandiflorum plays a significant role in the treatment of lung cancer patients ${ }^{[9]}$. In TCM, it is believed that Platycodon grandiflorum is the guide herb of the lung, which means that it serves as a boat of other drugs carrying them targeted to the lung. Despite the absence of study on the effect of Platycodon gandiflorum as single herb on lung, the research of Platycodon grandiflorum Decoction prescription which composes of Platycodon grandiflorum and GanCao (Glycyrrhiza Uralensis Fisch) found that it can effectively inhibit lung injury and inflammation $[10,11]$.

Similar to other TCM herbs, Platycodon grandiflorum is characterized by "multi-compound, multi-target, and multi-pathway", which makes it difficult to observe the effect of drugs on disease systematically and comprehensively. Network pharmacology as a new 
subject, has been accepted as an effective strategy to reveal the complicated correlation between TCM and diseases ${ }^{[12,13]}$. It relies on plenty of databases to screen the active compounds of TCM herbs, predict their target proteins, perform pathway enrichment and gene ontology enrichment for analyzing the mechanism of their treatment on a molecular level ${ }^{[14]}$.

In this work, we used network pharmacology theory to research the molecular mechanism of Platycodon grandiflorum in the treatment of lung cancer. The methodological framework of this study is shown in Fig. 1.

\section{Materials And Methods}

\subsection{Screening Active Compounds}

All of the chemical compounds were sourced from the Traditional Chinese Medicine Systems Pharmacology Database and Analysis Platform (TCMSP, http://tcmspw.com/tcmsp.php. Accessed 6 Mar 2020) ${ }^{[15]}$. In order to obtain active chemical compounds Oral Bioavailability $(O B \geq 30 \%)$ and Drug Likeness ( $D L \geq 0.10)$ were applied. OB revealed the convergence of the ADME process. The higher the $\mathrm{OB}$, the better the properties of bioactive molecules as therapeutic agents ${ }^{[16]}$. DL was taken as the standard used to evaluate the "drug like" degree of the prospective compounds. The value of DL was 0.18 , which could be treated as the screening criterion of TCM ${ }^{[17]}$.

\subsection{Predicting Compounds Targets and Construct Compounds-Targets Network}

In order to predict the target proteins of the compounds, we got the SMILEs and 3D structures of the compounds in PubChem (PubChem, https://pubchem.ncbi.nlm.nih.gov. Accessed 6 Mar 2020) [18], the world's largest collection of chemical information. The target proteins of active compounds were predicted in SwissTargetPrediction [19] (http://www.swisstargetprediction.ch. Accessed 6

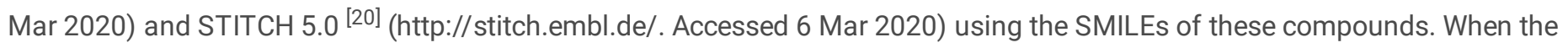
probability of obtained target proteins in SwissTargetPrediction, was greater than 0.5 could the subsequent analysis can be conducted. If the combined score of the obtained target protein in STITCH exceeded 0.7 , it could be included in the analysis. At the same time, the compound targets in the TCMSP were sorted out. After construction of a two-list table of compounds and targets, Cytoscape ${ }^{[21]}$ (Version. 3.7.1) was used to visualize the two-list table in a compounds-targets network. All targets were converted and included into UniProt ID by UniProt ${ }^{[22]}$ (UniProt, https://www.uniprot.org. Accessed 6 Mar 2020) for subsequent analysis.

\subsection{Screening of Protein Targets of Lung Cancer}

The keyword lung cancer was inputted into Therapeutic Target Database ${ }^{[23]}$ (TTD, http://db.idrblab.net/ttd/. Accessed 6 Mar 2020) and DisGeNET ${ }^{[24]}$ (https://www.disgenet.org. Accessed 6 Mar 2020). When the targets obtained from the TTD database were successfully verified, they could be included in the next analysis. Then, the targets were converted into UniProt ID by UniProt. The targets obtained from DisGeNET were screened, leaving the targets with UniProt ID and evidence index $(E I)=1$. The El showed conflicting results that supported the gene-disease associations (GDAs)/variant-disease associations (VDAs) in published publications. El = 1 indicates that all the publications support the GDAs or the VDAs.

\subsection{Mapping of Compounds Targets and Disease Targets and Constructing Protein-Protein Interaction (PPI) Network}

The disease targets and compounds targets were mapped with Venn figure in the Omicshare (https://www.omicshare.com/tools/. Accessed 7 Mar 2020) to find their common targets for further analysis. STRING $11.0^{[25]}$ (https://string-db.org/. Accessed 7 Mar 2020) was used to constructed PPI network for common targets under the default mode. The PPI network was inputted into Cytoscape for visualization.

\subsection{Screening Hub Targets}

The NetworkAnalayzer tool of Cytoscape was employed to analyze the topology of the imported network. The Betweenness centrality was intended to describe the centrality of nodes while Degree was purposed to described connectivity of the nodes. The targets with Degree and Betweenness Centrality greater than twice the median was defined as the hub targets. In order to clarify the expression differences of the 7 hub targets in lung cancer the data series of GEO database were searched out to and analyzed. 
DAVID Bioinformatics Resources $6.8^{\text {[26] }}$ (DAVID, https://david.ncifcrf.gov. Accessed 7 Mar 2020) was used to do GO and KEGG pathway Enrichment $(P<0.05)$. In order to show the relationship between compounds, targets and pathways in a more intuitive way, we used Cytoscape to construct compounds-targets-pathways network based on the enrichment results.

\subsection{Molecular Docking}

To verify our results, molecular docking was conducted to evaluate the binding strength of selected hub targets and their compounds. The mol2 file of the compounds in TCMSP and pdb file of the hub targets in Protein Data Bank ${ }^{[27]}$ (PDB, http://www.rcsb.org. Accessed 11 May 2020) were obtained. The selected protein structure exhibited the following three characteristics: X-ray diffraction structure, resolution $<2 \AA$, and had unique ligands. The crystal structure of protein was obtained after removing the solvent and organic by PyMOL software. Subsequent to the initial preparation by AutoDockTools ${ }^{[28]}$, the molecular docking was carried out with AutoVina ${ }^{[29]}$. In order to analyze the results of molecular docking, we analyzed the binding site of compounds ligands to targets proteins by PyMOL and analyzed the interaction of protein ligands by LigPlot ${ }^{\text {[30] }}$.

\section{Results}

\subsection{Active Chemical Compounds}

We obtained 102 compounds from the TCMSP. According to the OB and DL standards set there were as few as 7 compounds left after our screening. As the SMILEs could not be found in PubChem, 2-0-methyl-3-0- 3 -D-glucopyranosyl platycogenate $A$ and dimethyl 2-0-methyl-3-0-a-D-glucopyranosyl platycogenate A were excluded from the next analysis. In the following target prediction, Robinin had no effective protein target, so it was not included in the study. Finally, 4 compounds were analyzed. The basic information of these 4 compounds was shown in Table 1.

\subsection{Construction of compounds-targets network}

After the targets obtained from the TCMSP, SwissTargetPrediction and STITCH were sort out, we obtained 24 targets in the TCMSP, 42 targets in the SwissTargetPrediction and 24 targets in the STITCH. In total, 123 compounds targets were obtained, after deleting duplicates. The compounds-targets network was constructed used Cytoscape. The cluster analysis of the clusterMaker of Cytoscape led to four clusters, centered on four active compounds being obtained. Luteolin and Acacetin clusters contained more targets than the other two. The result was showed in Fig. 2.

\subsection{The Targets of Lung Cancer}

A total of 45 lung cancer related genes were identified in TTD database. There were three groups of disease data selected from DisGeNET with the Disease ID of C0684249, C0242379 and C0007121. In these three sets of data, 1963, 1991 and 37 target genes were obtained respectively. After removing the repeats, we finally got 2129 disease targets.

\subsection{The Common Target of Compounds and Diseases and PPI Network}

Venn figure was used to identify the common targets of compounds and diseases. According to the result, 80 targets were shared between them, among which the targets from Luteolin were the most,accounting for 64 . Apart from that, 22 targets form Acacetin, 7 targets form cis-Dihydroquercetin, and 2 targets form Spinasterol. The result was showed in Fig. 3A. The 80 targets as mentioned above were inputted into String database to get a PPI network, which consisted 80 nodes $\square$ and 1057 edges. We used Cytoscape to visualize and analyze the PPI network. The result was showed in Fig. 3B.

\subsection{Hub Targets}

After the visualization of the PPI network by Cytoscape and the topological analysis of the Network Analayzer tool, the details of the topological indexes such as Degree and Betweenness Centrality were obtained. According to the hub target standard defined by us, the target with the Degree and the Betweenness Centrality more than twice the median was identified as the hub target. Based on the stander 7 hub targets were identified. The topological indexes of 7 hub targets were showed in table 2 . 
In order to clarify the expression differences of the 7 hub targets in lung cancer and normal lung tissues, we retrieved data series GSE19804 in the GEO database (https://www.ncbi.nlm.nih.gov/geo/query/acc.cgi?acc=GSE19804. Accessed 23 May 2020) ${ }^{[31]}$. We obtained 120 samples from paired tumor and normal tissues. We used the heatmap.2 package of $\mathrm{R}$ to normalize the data and draw the heatmap. We found that the expression of AKT1, CASP3, EGFR and TP53 was up-regulated and the expression of IL-6, MAPK1 and VEGFA was down-regulated in lung cancer. The expression level of each gene was shown by scatter plot. The results were showed in Fig. 4.

\subsection{GO Enrichment}

David was applied to do GO Enrichment of the 7 hub targets and then 81 enrichment results were obtained, including 67 biological processes (BP), 10 molecular functions (MF), and 4 cellular components (CC). The top ten go enrichment results of each group were showed in Fig. 5.

\subsection{KEGG Pathway Enrichment}

David was applied to carry out KEGG pathway Enrichment of the 7 hub targets. After removing the extensive pathways, 23 pathways were obtained. The top three were Proteoglycans in cancer, HIF - 1 signaling pathway and PI3K - Akt signaling pathway. The result of the 23 pathways was showed in Fig. 6.

Then, constructed compounds-targets-pathways network was constructed with the 7 hub targets. A network with 32 nodes and 114 edges was obtained, including 2 compounds, 7 targets, 23 pathways, 21 targets-targets interaction, 10 compounds-targets interaction and 83 targets-pathways interaction. After modular analysis the result of was showed in Fig. 7.

\subsection{Molecular Docking Result}

AutoVina was employed to conducted molecular docking, with the result listed in table 3 . The reliability of the docking results was evaluated against with the score of Affinity. The value of affinity represented the strength of binding between the compounds and the targets. The greater the negative value, the more satisfactory the binding result. The best binding protein of compound Luteolin was 5UG9, the structure of EGFR. The best binding protein of compound Acacetin was 1NME, the structure of CASP3. Their threedimensional structure and protein ligand interaction was showed in Fig. 8.

\section{Discussion}

This study explored the pharmacological mechanisms of Platycodon grandiflorum in the treatment of lung cancer in a systematic way through network pharmacology analysis. There were four active compounds screened out, including Acacetin, Spinasterol, cisDihydroquercetin and Luteolin. Acacetin was a kind of flavonoid compounds, had anti-cancer, anti-inflammatory and antioxidative activities. It could not only inhibit the invasion and migration of human non-small cell lung cancer (NSCLC) A549 cells and human prostate cancer DU145 cells ${ }^{[32,33]}$, but also induced mitochondrial ROS-mediated colon carcinoma cells death by inducing apoptosis inducing factor ${ }^{[34]}$. These results showed that Acacetin had anti-cancer effect. Spinasterol was a kind of natural phytoestrogen, which can be used as a chemical preventive agent for some malignant tumors. It had a strong anti-proliferation effect on breast cancer, ovarian cancer, cervical cancer cells ${ }^{[35-37]}$. Dihydroquercetin was a botanical antioxidant capable to protect from the damage caused by oxidative ${ }^{[38,39]}$. Luteolin was also a natural flavonoid compound, which possessed antioxidant, antiinflammation and anti-cancer properties. It was clear that Luteolin could inhibit inflammation. In addition, it could inhibit acute lung injury inflammation by regulating the pro-inflammatory medium HMGB1 ${ }^{[40]}$. The anti-cancer effect of Luteolin was realized by direct inhibition cancer cells and chemo-sensitizing effect. Firstly, Luteolin inhibited lung cancer by inducing G1 phase cell cycle arrest and apoptosis as well as through the down regulation of AIM2 [41, 42]. Secondly, Luteolin had the chemo-sensitizing effect, which has been proven in gastric cancer and ovarian cancer ${ }^{[43,44]}$. It was found out that the four compounds screened by us either produced anti-cancer effect or inhibited lung injury inflammation.

The 7 hub targets, we got in this study, were all closely associated with lung cancer. It was found that the over-expression of AKT1 stimulated the proliferation of lung cancer cells, and the inhibition of AKT1 reduced the proliferation and migration of lung cancer cells ${ }^{[45-47]}$. The high level of AKT1 expression was related to the drug resistance of lung cancer cells ${ }^{[48]}$. The polymorphism of 
CASP3 gene related to apoptosis was associated with lung cancer susceptibility, and CASP3 played a major role in cell apoptosis and carcinogenesis ${ }^{[49]}$. We found that the $1 \mathrm{NME}$, a crystal structure of target CASP3 could have intense interaction with compound Acacetin. EGFR mutation played a major role in the carcinogenesis of NSCL ${ }^{[50,51]}$. EGFR could be transactivated by PAR2 receptor to achieve the transfer activity of A549 cells ${ }^{[52]}$. In previous studies, the drug resistance caused by EGFR-C797S mutations accounted for about a quarter of the sample ${ }^{[53]}$. According to our molecular docking results, the compound Luteolin and 5UG9, the crystal structure of target EGFR, performed best in binding degree. It was speculated that EGFR might been one of the key targets of Platycodon grandiflorum. The mutation of TP53 might damage the function of lung epithelium, changed the pulmonary microbial community, and allowed tumor foraging bacteria to proliferate ${ }^{[54]}$. TP53 stimulated apoptosis of NSCLC cells and lung cancer stem cells ${ }^{[55]}$. TP53 mutation played an important role in the prognosis of lung cancer ${ }^{[56]}$. TP53 mutation was a negative prognostic factor for overall survival ${ }^{[57]}$. The activation of IL-6/STAT3 pathway could accelerate the migration and invasion of lung cancer cells by promoting the epithelial mesenchymal transformation ${ }^{[58]}$. IL-6 blockade could significantly inhibit lung cancer ${ }^{[59]}$. In the meantime, the activated IL-6/STAT3 signal mediated the drug resistance ${ }^{[60]}$. Our statistical results showed that the expression of IL6 was low in lung cancer tissues, which was different from the results of literature. This might be related to the source of samples we analyzed. The expression of IL- 6 was closely related to smoking. The samples we analyzed were non-smoking female, and there was a certain deviation in the results. And literature studies found that $47 \%$ of lung cancer samples had high expression of IL-6 ${ }^{[61]}$, increased IL- 6 levels were only associated with lung cancer diagnosed within 2 years of blood collection ${ }^{[62]}$. MAPK1, also known as ERK2, the expression of MAPK1 was down-regulated which could interfere the apoptosis of lung cancer cells ${ }^{[63]}$. VEGF was effective in promoting tumorigenesis by vascular remodeling. Regulating the level of VEGF and inhibiting angiogenesis, the malignant progression of lung cancer can be partly inhibited ${ }^{[64]}$. The expression of VEGFA were controversial. In adenocarcinoma but not in squamous cell carcinoma, VEGFA protein levels were significantly associated with the tumor size and lymph node metastasis ${ }^{[65]}$. This explains why VEGFA was low expression in the data GSE19804 which we analyzed and high expression in lung adenocarcinoma samples GSE10072 (https://www.ncbi.nlm.nih.gov/geo/query/acc.cgi?acc=GSE10072. Accessed 23 May 2020).

For further analysis of the pathways enriched, we searched the Kyoto Encyclopedia of Genes and Genomes (KEGG, https://www.genome.jp/kegg/. Accessed 25 Mar 2020). We found that PI3K-Akt signaling pathway and MAPK signaling pathway had a potential to play a central role in Platycodon grandiflorum regulation. The PI3K-Akt signaling pathway could regulates such primary cellular functions as transcription, translation, proliferation, growth, and survival. The MAPK signaling pathway involved in various cellular functions, for example cell proliferation, differentiation and migration. We found that TNF signaling pathway, FoxO signaling pathway, Toll-like receptor signaling pathway, Thyroid hormone signaling pathway, Neurotrophin signaling pathway, NODlike receptor signaling pathway, ErbB signaling pathway, Ras signaling pathway, Rap1 signaling pathway, VEGF signaling pathway and Choline metabolism in cancer were all upstream or downstream of PI3K-Akt and MAPK signaling pathways. HIF-1 signaling pathway could activate VEGF signaling pathway, which was indirectly associated with them. HIF-1 transcription factor was also one of the regulators for tumor metabolism as well and affected the Central carbon metabolism of cancer. There was also a complex relationship found between Choline metabolism in cancer and HIF-1, Ras, PI3K. Moreover, Platycodon grandiflorum might also play a role in regulating the apoptosis of lung cancer, changing the proteoglycan of tumor microenvironment, and regulating the autophagy of tumor cells.

\section{Conclusion}

As revealed by our literature search and network pharmacological analysis, Platycodon grandiflorum was effective in the treatment of lung cancer. It is capable of regulating various biological processes such as cell transcription, translation, proliferation, apoptosis, migration and so on. Though its effect was the result of the regulation of multiple pathways, our study still led to the finding that PI3K-Akt signal pathway and MAPK signal pathway may be the most significant pathways. According to our clinical experience, Platycodon grandiflorum, as a guide herb of the lung, might play a particularly significant role on pre activate of the targets above, which will improve the efficacy of other anticancer drugs. The next experiment needs us to carry out further.

\section{Declarations}

Funding 
We appreciate the financial support received from the National Natural Science Foundation of China (81573728), National Science and Technology Major Project (2017ZX09301030).

\section{Availability of data and materials}

The datasets used and/or analyzed during the study are available from the corresponding author on reasonable request.

\section{Ethics approval and consent to participate}

Not applicable.

\section{Consent for publication}

The manuscript is approved by all authors for publication.

\section{Competing interests}

The authors declare that they have no competing interests.

\section{Authors' contributions}

Ren-Zhong Wang and Cheng-Fang Yao designed and conducted the study with equal contribution. Xiang-Jing Chen and Ran Zhang collected the data and wrote the paper. All authors read and approved the final manuscript.

\section{Acknowledgements}

Not applicable.

\section{References}

1. Bray F, Ferlay J, Soerjomataram I, Siegel RL, Torre LA, Jemal A. Global cancer statistics 2018: GLOBOCAN estimates of incidence and mortality worldwide for 36 cancers in 185 countries. CA Cancer J Clin. 2018;68(6):394-424.

2. Ettinger DS, Aisner DL, Wood DE, Akerley W, Bauman J, Chang JY, Chirieac LR, D'Amico TA, Dilling TJ, Dobelbower M, et al. NCCN Guidelines Insights: Non-Small Cell Lung Cancer, Version 5.2018. J Natl Compr Canc Netw. 2018;16(7):807-21.

3. Kalemkerian GP, Loo BW, Akerley W, Attia A, Bassetti M, Boumber Y, Decker R, Dobelbower MC, Dowlati A, Downey RJ, et al. NCCN Guidelines Insights: Small Cell Lung Cancer, Version 2.2018. J Natl Compr Canc Netw. 2018;16(10):1171-82.

4. Yang W, Kang Y, Zhao Q, Bi L, Jiao L, Gu Y, Lu J, Yao J, Zhou D, Sun J, et al. Herbal formula Yangyinjiedu induces lung cancer cell apoptosis via activation of early growth response 1. J Cell Mol Med. 2019;23(9):6193-202.

5. Liao YH, Li Cl, Lin CC, Lin JG, Chiang JH, Li TC. Traditional Chinese medicine as adjunctive therapy improves the long-term survival of lung cancer patients. J Cancer Res Clin Oncol. 2017;143(12):2425-35.

6. Jiao L, Dong C, Liu J, Chen Z, Zhang L, Xu J, Shen X, Che J, Yang Y, Huang H, et al. Effects of Chinese Medicine as Adjunct Medication for Adjuvant Chemotherapy Treatments of Non-Small Cell Lung Cancer Patients. Sci Rep. 2017;7:46524.

7. Guo H, Liu JX, Li H, Baak JPA. In Metastatic Non-small cell Lung Cancer Platinum-Based Treated Patients, Herbal Treatment Improves the Quality of Life. A Prospective Randomized Controlled Clinical Trial. Front Pharmacol. 2017;8:454.

8. Li TM, Yu YH, Tsai FJ, Cheng CF, Wu YC, Ho TJ, Liu X, Tsang H, Lin TH, Liao CC, et al. Characteristics of Chinese herbal medicine usage and its effect on survival of lung cancer patients in Taiwan. J Ethnopharmacol. 2018;213:92-100.

9. Lin Y-J, Liang W-M, Chen C-J, Tsang H, Chiou J-S, Liu X, Cheng C-F, Lin T-H, Liao C-C, Huang S-M, et al: Network analysis and mechanisms of action of Chinese herb-related natural compounds in lung cancer cells. Phytomedicine 2019, 58.

10. Tao J, Nie Y, Hou Y, Ma X, Ding G, Gao J, Jiang M, Bai G. Chemomics-Integrated Proteomics Analysis of Jie-Geng-Tang to Ameliorate Lipopolysaccharide-Induced Acute Lung Injury in Mice. Evidence-Based Complementary Alternative Medicine. 2016;2016:1-12. 
11. Liu Y, Hong Z, Qian J, Wang Y, Wang S. Protective effect of Jie-Geng-Tang against Staphylococcus aureus induced acute lung injury in mice and discovery of its effective constituents. J Ethnopharmacol. 2019;243:112076.

12. Sun JH, Sun F, Yan B, Li JY, Xin L. Data mining and systematic pharmacology to reveal the mechanisms of traditional Chinese medicine in Mycoplasma pneumoniae pneumonia treatment. Biomed Pharmacother. 2020;125:109900.

13. Su X, Li Y, Jiang M, Zhu J, Zheng C, Chen X, Zhou J, Li Y, Xiao W, Wang Y. Systems pharmacology uncover the mechanism of anti-non-small cell lung cancer for Hedyotis diffusa Willd. Biomed Pharmacother. 2019;109:969-84.

14. Li X, Yang H, Xiao J, Zhang J, Zhang J, Liu M, Zheng Y, Ma L. Network pharmacology based investigation into the bioactive compounds and molecular mechanisms of Schisandrae Chinensis Fructus against drug-induced liver injury. Bioorganic Chemistry 2020, 96.

15. Ru J, Li P, Wang J, Zhou W, Li B, Huang C, Li P, Guo Z, Tao W, Yang Y, et al. TCMSP: a database of systems pharmacology for drug discovery from herbal medicines. J Cheminform. 2014;6:13.

16. Xu X, Zhang W, Huang C, Li Y, Yu H, Wang Y, Duan J, Ling Y. A novel chemometric method for the prediction of human oral bioavailability. Int J Mol Sci. 2012;13(6):6964-82.

17. Tao W, Xu X, Wang X, Li B, Wang Y, Li Y, Yang L. Network pharmacology-based prediction of the active ingredients and potential targets of Chinese herbal Radix Curcumae formula for application to cardiovascular disease. J Ethnopharmacol. 2013;145(1):1-10.

18. Bolton EE, Chen J, Kim S, Han L, He S, Shi W, Simonyan V, Sun Y, Thiessen PA, Wang J, et al. PubChem3D: a new resource for scientists. J Cheminform. 2011;3(1):32.

19. Daina A, Michielin O, Zoete V. SwissTargetPrediction: updated data and new features for efficient prediction of protein targets of small molecules. Nucleic Acids Res. 2019;47(W1):W357-w364.

20. Szklarczyk D, Santos A, von Mering C, Jensen LJ, Bork P, Kuhn M. STITCH 5: augmenting protein-chemical interaction networks with tissue and affinity data. Nucleic Acids Res. 2016;44(D1):D380-4.

21. Shannon P, Markiel A, Ozier O, Baliga NS, Wang JT, Ramage D, Amin N, Schwikowski B, Ideker T. Cytoscape: a software environment for integrated models of biomolecular interaction networks. Genome Res. 2003;13(11):2498-504.

22. Consortium U. UniProt: a worldwide hub of protein knowledge. Nucleic acids research. 2019;47:D506-15.

23. Wang Y, Zhang S, Li F, Zhou Y, Zhang Y, Wang Z, Zhang R, Zhu J, Ren Y, Tan Y, et al. Therapeutic target database 2020: enriched resource for facilitating research and early development of targeted therapeutics. Nucleic Acids Res. 2020;48(D1):D1031-d1041.

24. Pinero J, Ramirez-Anguita JM, Sauch-Pitarch J, Ronzano F, Centeno E, Sanz F, Furlong LI. The DisGeNET knowledge platform for disease genomics: 2019 update. Nucleic Acids Res. 2020;48(D1):D845-d855.

25. Szklarczyk D, Franceschini A, Kuhn M, Simonovic M, Roth A, Minguez P, Doerks T, Stark M, Muller J, Bork P, et al. The STRING database in 2011: functional interaction networks of proteins, globally integrated and scored. Nucleic Acids Res. 2011;39(Database issue):D561-8.

26. Huang da W, Sherman BT, Lempicki RA. Systematic and integrative analysis of large gene lists using DAVID bioinformatics resources. Nat Protoc. 2009;4(1):44-57.

27. Berman H, Henrick K, Nakamura H. Announcing the worldwide Protein Data Bank. Nature structural biology. 2003;10(12):980.

28. Morris GM, Huey R, Lindstrom W, Sanner MF, Belew RK, Goodsell DS, Olson AJ. AutoDock4 and AutoDockTools4: Automated docking with selective receptor flexibility. J Comput Chem. 2009;30(16):2785-91.

29. Trott O, Olson AJ. AutoDock Vina: improving the speed and accuracy of docking with a new scoring function, efficient optimization, and multithreading. J Comput Chem. 2010;31(2):455-61.

30. Wallace AC, Laskowski RA, Thornton JM. LIGPLOT: a program to generate schematic diagrams of protein-ligand interactions. Protein engineering. 1995;8(2):127-34.

31. Barrett T, Wilhite SE, Ledoux P, Evangelista C, Kim IF, Tomashevsky M, Marshall KA, Phillippy KH, Sherman PM, Holko M, et al. NCBI GEO: archive for functional genomics data sets-update. Nucleic Acids Res. 2013;41(Database issue):D991-5.

32. Chien ST, Lin SS, Wang CK, Lee YB, Chen KS, Fong Y, Shih YW. Acacetin inhibits the invasion and migration of human non-small cell lung cancer A549 cells by suppressing the p38alpha MAPK signaling pathway. Mol Cell Biochem. 2011;350(1-2):135-48.

33. Shen KH, Hung SH, Yin LT, Huang CS, Chao CH, Liu CL, Shih YW. Acacetin, a flavonoid, inhibits the invasion and migration of human prostate cancer DU145 cells via inactivation of the p38 MAPK signaling pathway. Mol Cell Biochem. 2010;333(1- 
2):279-91.

34. Prasad N, Sharma JR, Yadav UCS. Induction of growth cessation by acacetin via beta-catenin pathway and apoptosis by apoptosis inducing factor activation in colorectal carcinoma cells. Mol Biol Rep. 2020;47(2):987-1001.

35. Jeon GC, Park MS, Yoon DY, Shin CH, Sin HS, Um SJ. Antitumor activity of spinasterol isolated from Pueraria roots. Exp Mol Med. 2005;37(2):111-20.

36. SE M-S, M N-N, E R-B, CL D-T-S, M J-E, RE R-Z. Stegnosperma halimifoliumAntiproliferative activity of spinasterol isolated of (Benth, 1844). Saudi pharmaceutical journal: SPJ : the official publication of the Saudi Pharmaceutical Society. 2017;25(8):1137-43.

37. Sedky NK, El Gammal ZH, Wahba AE, Mosad E, Waly ZY, El-Fallal AA, Arafa RK, El-Badri N. The molecular basis of cytotoxicity of alpha-spinasterol from Ganoderma resinaceum: Induction of apoptosis and overexpression of p53 in breast and ovarian cancer cell lines. J Cell Biochem. 2018;119(5):3892-902.

38. Zai W, Chen W, Luan J, Fan J, Zhang X, Wu Z, Ding T, Ju D, Liu H. Dihydroquercetin ameliorated acetaminophen-induced hepatic cytotoxicity via activating JAK2/STAT3 pathway and autophagy. Appl Microbiol Biotechnol. 2018;102(3):1443-53.

39. Zivkovic L, Bajic V, Topalovic D, Bruic M, Spremo-Potparevic B: Antigenotoxic Effects of Biochaga and Dihydroquercetin (Taxifolin) on H2O2-Induced DNA Damage in Human Whole Blood Cells. Oxid Med Cell Longev 2019, 2019:5039372.

40. Park EJ, Kim YM, Kim HJ, Chang KC. Luteolin activates ERK1/2- and $\mathrm{Ca}(2+)$-dependent HO-1 induction that reduces LPS-induced HMGB1, iNOS/NO, and COX-2 expression in RAW264.7 cells and mitigates acute lung injury of endotoxin mice. Inflamm Res. 2018;67(5):445-53.

41. Zhao Y, Yang G, Ren D, Zhang X, Yin Q, Sun X. Luteolin suppresses growth and migration of human lung cancer cells. Mol Biol Rep. 2011;38(2):1115-9.

42. Yu Q, Zhang M, Ying Q, Xie X, Yue S, Tong B, Wei Q, Bai Z, Ma L. Decrease of AIM2 mediated by luteolin contributes to non-small cell lung cancer treatment. Cell Death Dis. 2019;10(3):218.

43. Wu B, Zhang Q, Shen W, Zhu J. Anti-proliferative and chemosensitizing effects of luteolin on human gastric cancer AGS cell line. Mol Cell Biochem. 2008;313(1-2):125-32.

44. Wang H, Luo Y, Qiao T, Wu Z, Huang Z. Luteolin sensitizes the antitumor effect of cisplatin in drug-resistant ovarian cancer via induction of apoptosis and inhibition of cell migration and invasion. J Ovarian Res. 2018;11(1):93.

45. Zhang G, Chen HX, Yang SN, Zhao J. MAGI1-IT1 stimulates proliferation in non-small cell lung cancer by upregulating AKT1 as a ceRNA. Eur Rev Med Pharmacol Sci. 2020;24(2):691-8.

46. Yoo JK, Jung HY, Lee JM, Yi H, Oh SH, Ko HY, Yoo H, Kim HR, Song H, Kim S, et al. The novel miR-9500 regulates the proliferation and migration of human lung cancer cells by targeting Akt1. Cell Death Differ. 2014;21(7):1150-9.

47. Rao G, Pierobon M, Kim IK, Hsu WH, Deng J, Moon YW, Petricoin EF, Zhang YW, Wang Y, Giaccone G. Inhibition of AKT1 signaling promotes invasion and metastasis of non-small cell lung cancer cells with K-RAS or EGFR mutations. Sci Rep. 2017;7(1):7066.

48. Liu LZ, Zhou XD, Qian G, Shi X, Fang J, Jiang BH. AKT1 amplification regulates cisplatin resistance in human lung cancer cells through the mammalian target of rapamycin/p70S6K1 pathway. Cancer Res. 2007;67(13):6325-32.

49. Lin J, Zhang Y, Wang H, Chang J, Wei L, Cao L, Zhang Z, Zhang X. Genetic Polymorphisms in the Apoptosis-Associated Gene CASP3 and the Risk of Lung Cancer in Chinese Population. PLoS One. 2016;11(10):e0164358.

50. Schmid S, Fruh M, Peters S. Targeting MET in EGFR resistance in non-small-cell lung cancer-ready for daily practice? Lancet Oncol. 2020;21(3):320-2.

51. Harrison PT, Vyse S, Huang PH. Rare epidermal growth factor receptor (EGFR) mutations in non-small cell lung cancer. Semin Cancer Biol. 2020;61:167-79.

52. Kim GT, Hahn KW, Yoon SY, Sohn KY, Kim JW. PLAG Exerts Anti-Metastatic Effects by Interfering with Neutrophil Elastase/PAR2/EGFR Signaling in A549 Lung Cancer Orthotopic Model. Cancers 2020, 12(3).

53. Lee J, Kim HS, Lee B, Kim HK, Sun JM, Ahn JS, Ahn MJ, Park K, Lee SH. Genomic landscape of acquired resistance to thirdgeneration EGFR tyrosine kinase inhibitors in EGFR T790M-mutant non-small cell lung cancer. Cancer 2020.

54. Greathouse KL, White JR, Vargas AJ, Bliskovsky VV, Beck JA, von Muhlinen N, Polley EC, Bowman ED, Khan MA, Robles Al, et al. Interaction between the microbiome and TP53 in human lung cancer. Genome Biol. 2018;19(1):123. 
55. Wang M, Wang X, Li Y, Xiao Q, Cui XH, Xiao GD, Wang JC, Xu CW, Ren H, Liu D. Nutlin-3-Induced Sensitization of Non-Small Cell Lung Cancer Stem Cells to Axitinib-Induced Apoptosis Through Repression of Akt1/Wnt Signaling. Oncol Res. 2019;27(9):98795.

56. Costa DB. TP53 mutations are predictive and prognostic when co-occurring with ALK rearrangements in lung cancer. Ann Oncol. 2018;29(10):2028-30.

57. Aisner DL, Sholl LM, Berry LD, Rossi MR, Chen H, Fujimoto J, Moreira AL, Ramalingam SS, Villaruz LC, Otterson GA, et al. The Impact of Smoking and TP53 Mutations in Lung Adenocarcinoma Patients with Targetable Mutations-The Lung Cancer Mutation Consortium (LCMC2). Clin Cancer Res. 2018;24(5):1038-47.

58. Lu YY, Lin Y, Ding DX, Su S, Chi QQ, Zhang YC, Sun J, Zhang X, Zhu HM, Huang QS, et al. MiR-26a functions as a tumor suppressor in ambient particulate matter-bound metal-triggered lung cancer cell metastasis by targeting LIN28B-IL6-STAT3 axis. Arch Toxicol. 2018;92(3):1023-35.

59. Caetano MS, Zhang H, Cumpian AM, Gong L, Unver N, Ostrin EJ, Daliri S, Chang SH, Ochoa CE, Hanash S, et al. IL6 Blockade Reprograms the Lung Tumor Microenvironment to Limit the Development and Progression of K-ras-Mutant Lung Cancer. Cancer Res. 2016;76(11):3189-99.

60. Yang Y, Wang W, Chang H, Han Z, Yu X, Zhang T. Reciprocal regulation of miR-206 and IL-6/STAT3 pathway mediates IL6induced gefitinib resistance in EGFR-mutant lung cancer cells. J Cell Mol Med. 2019;23(11):7331-41.

61. Koh E, lizasa T, Yamaji H, Sekine Y, Hiroshima K, Yoshino I, Fujisawa T. Significance of the correlation between the expression of interleukin 6 and clinical features in patients with non-small cell lung cancer. Int J Surg Pathol. 2012;20(3):233-9.

62. Pine SR, Mechanic LE, Enewold L, Chaturvedi AK, Katki HA, Zheng YL, Bowman ED, Engels EA, Caporaso NE, Harris CC. Increased levels of circulating interleukin 6, interleukin 8, C-reactive protein, and risk of lung cancer. J Natl Cancer Inst.

2011;103(14):1112-22.

63. Frese S, Pirnia F, Miescher D, Krajewski S, Borner MM, Reed JC, Schmid RA. PG490-mediated sensitization of lung cancer cells to Apo2L/TRAIL-induced apoptosis requires activation of ERK2. Oncogene. 2003;22(35):5427-35.

64. Liang L, Hui K, Hu C, Wen Y, Jiang X. Autophagy inhibition potentiates the anti-angiogenic property of multikinase inhibitor anlotinib through JAK2/STAT3/VEGFA signaling in non-small cell lung cancer cells. Ann Oncol. 2019;30(Suppl 2):ii4.

65. Qin S, Yi M, Jiao D, Li A, Wu K. Distinct Roles of VEGFA and ANGPT2 in Lung Adenocarcinoma and Squamous Cell Carcinoma. J Cancer. 2020;11(1):153-67.

\section{Tables}

Due to technical limitations, Table 1 is provided in the Supplementary Files section.

Table 2 Topological analysis of the 7 hub targets.

\begin{tabular}{|lllllllll|}
\hline Targets & Degree & $\begin{array}{l}\text { Betweenness } \\
\text { Centrality }\end{array}$ & $\begin{array}{l}\text { Average } \\
\text { Shortest } \\
\text { Path } \\
\text { Length }\end{array}$ & $\begin{array}{l}\text { Closeness } \\
\text { Centrality }\end{array}$ & $\begin{array}{l}\text { Clustering } \\
\text { Coefficient }\end{array}$ & $\begin{array}{l}\text { Eccentricity } \\
\text { Connectivity }\end{array}$ & $\begin{array}{l}\text { Neighborhood } \\
\text { Radiality }\end{array}$ \\
\hline TP53 & 64 & 0.0656612 & 1.18987342 & 0.84042553 & 0.43799603 & 2 & 30.21875 & 0.95253165 \\
\hline AKT1 & 64 & 0.06244503 & 1.18987342 & 0.84042553 & 0.43005952 & 2 & 29.9375 & 0.95253165 \\
\hline VEGFA & 59 & 0.05210755 & 1.25316456 & 0.7979798 & 0.46756283 & 2 & 31.15254237 & 0.93670886 \\
\hline CASP3 & 58 & 0.03274417 & 1.26582278 & 0.79 & 0.50030248 & 2 & 32.22413793 & 0.9335443 \\
\hline IL6 & 53 & 0.05505548 & 1.35443038 & 0.73831776 & 0.47605225 & 3 & 30.67924528 & 0.91139241 \\
\hline EGFR & 53 & 0.03692371 & 1.32911392 & 0.75238095 & 0.49419448 & 2 & 32.24528302 & 0.91772152 \\
\hline MAPK1 & 52 & 0.03580909 & 1.35443038 & 0.73831776 & 0.5188537 & 3 & 32.63461538 & 0.91139241 \\
\hline
\end{tabular}


Table 3 Molecular docking information

\begin{tabular}{|llll|}
\hline Compound & Target & PDB ID & $\begin{array}{l}\text { Affinity } \\
\text { (kal/mol) }\end{array}$ \\
\hline Luteolin & TP53 & $6 \mathrm{SHZ}$ & -8.6 \\
\hline Luteolin & AKT1 & 1UNQ & -7.1 \\
\hline Luteolin & VEGFA & $4 \mathrm{KZN}$ & -6.6 \\
\hline Luteolin & CASP3 & 1NME & -9.4 \\
\hline Luteolin & IL6 & 1ALU & -8.0 \\
\hline Luteolin & EGFR & $5 \mathrm{UG9}$ & -9.8 \\
\hline Luteolin & MAPK1 & $4 \mathrm{ZZN}$ & -8.4 \\
\hline Acacetin & TP53 & $6 \mathrm{SHZ}$ & -8.2 \\
\hline Acacetin & VEGFA & $4 \mathrm{KZN}$ & -6.5 \\
\hline Acacetin & CASP3 & 1NME & -9.1 \\
\hline
\end{tabular}

\section{Figures}




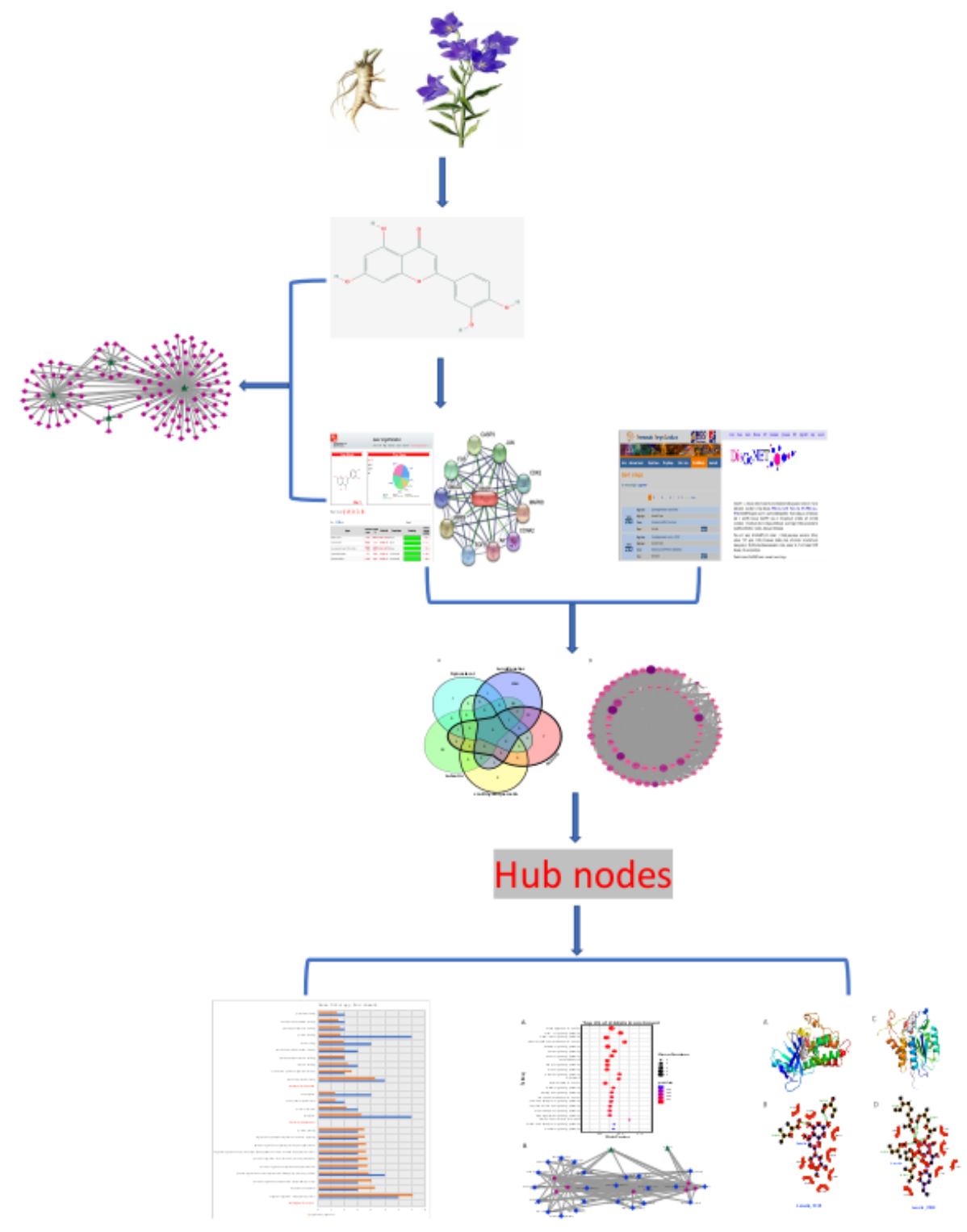

Figure 1

Technology roadmap. 


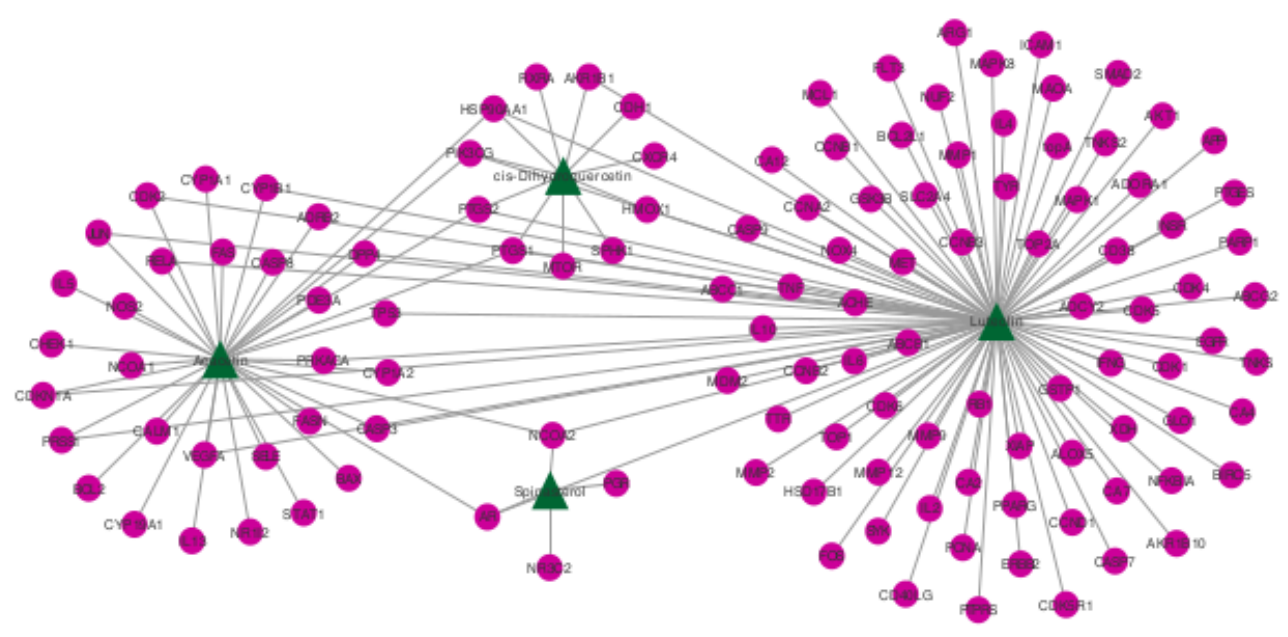

\section{Figure 2}

Compounds-Targets Network. Green triangle represented the compounds, red circle represented the targets.

A

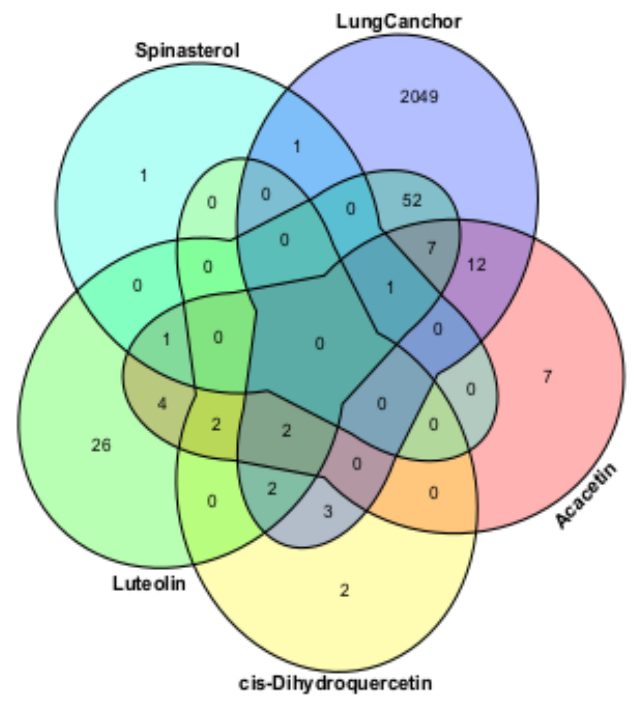

B

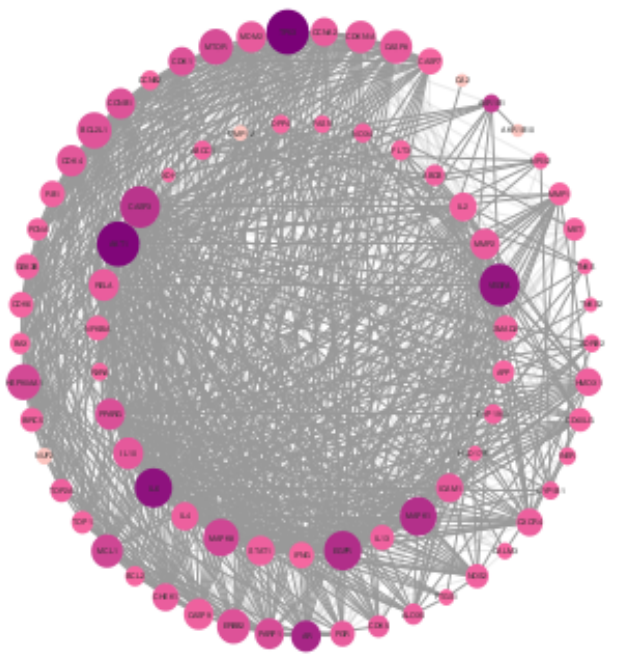

\section{Figure 3}

A. Venn figure of compound targets and disease targets. B. PPI Network. The protein-protein interaction of the 80 common targets of compounds and diseases. The darker the color was, the greater the Betweenness Centrality was, and the larger the circle was, the greater the Degree was. 


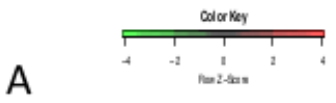

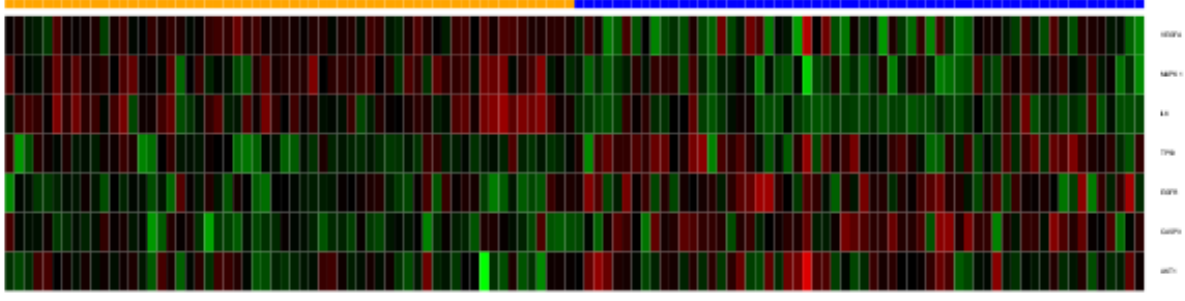

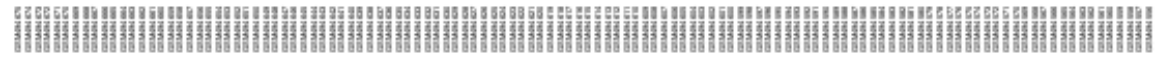

B
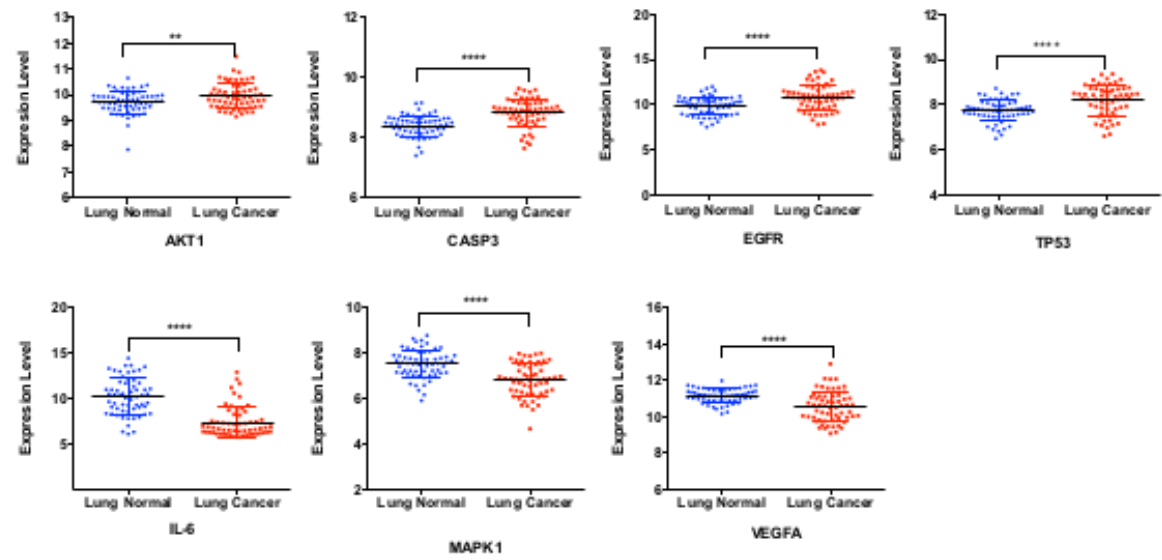

\section{Figure 4}

A. Heat map of hub targets. 60 lung cancer samples and 60 lung normal samples were showed. B. The expression level of hub targets. ${ }^{\star *} \mathrm{P}<0.01 .{ }^{* \star \star *} \mathrm{P}<0.0001$. 


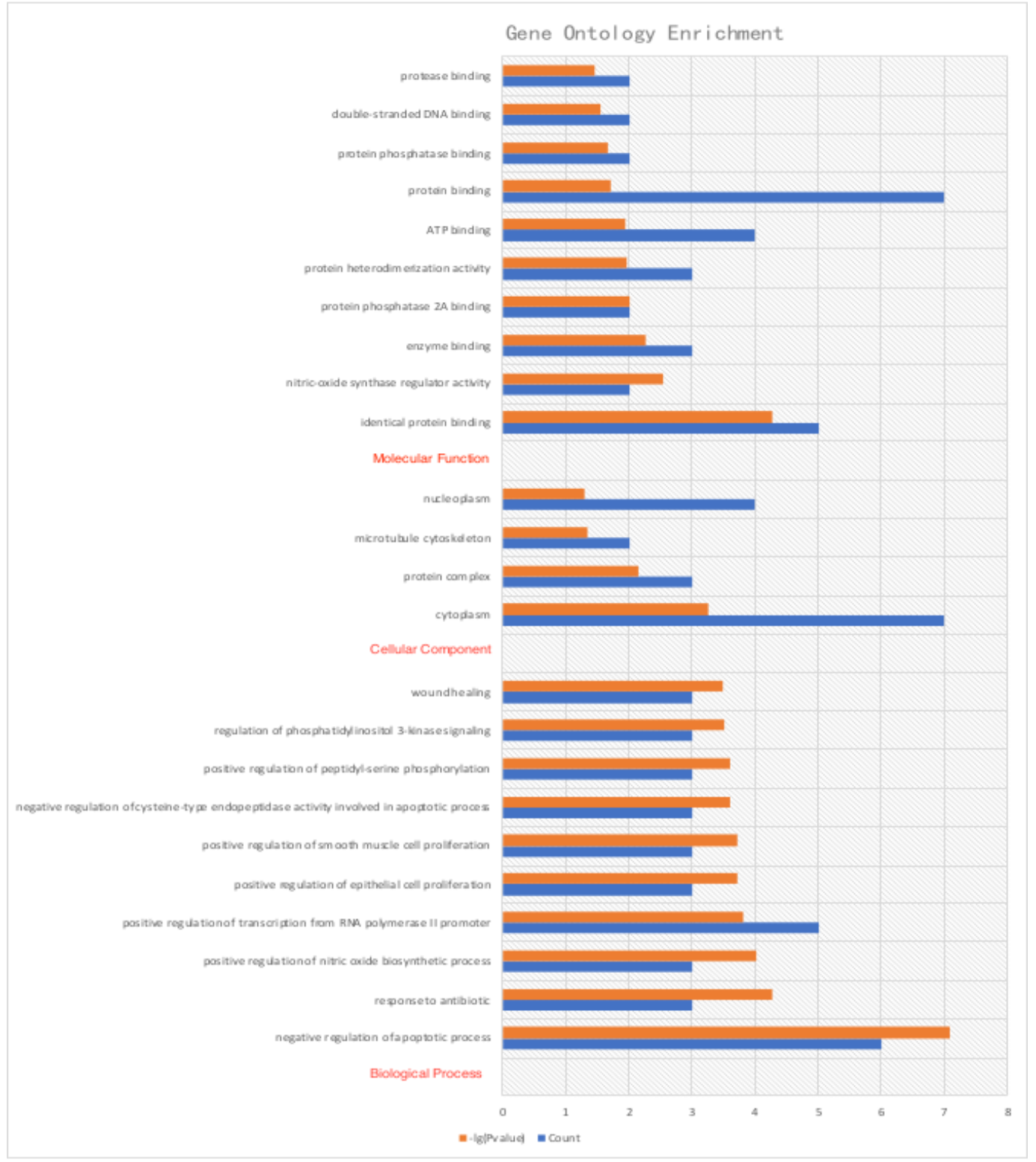

Figure 5

GO Enrichment. The top 10 results of BP, MF and CC. The height of the blue column represented the number of targets. The height of the red column represented $-\lg$ (Pvalue). 


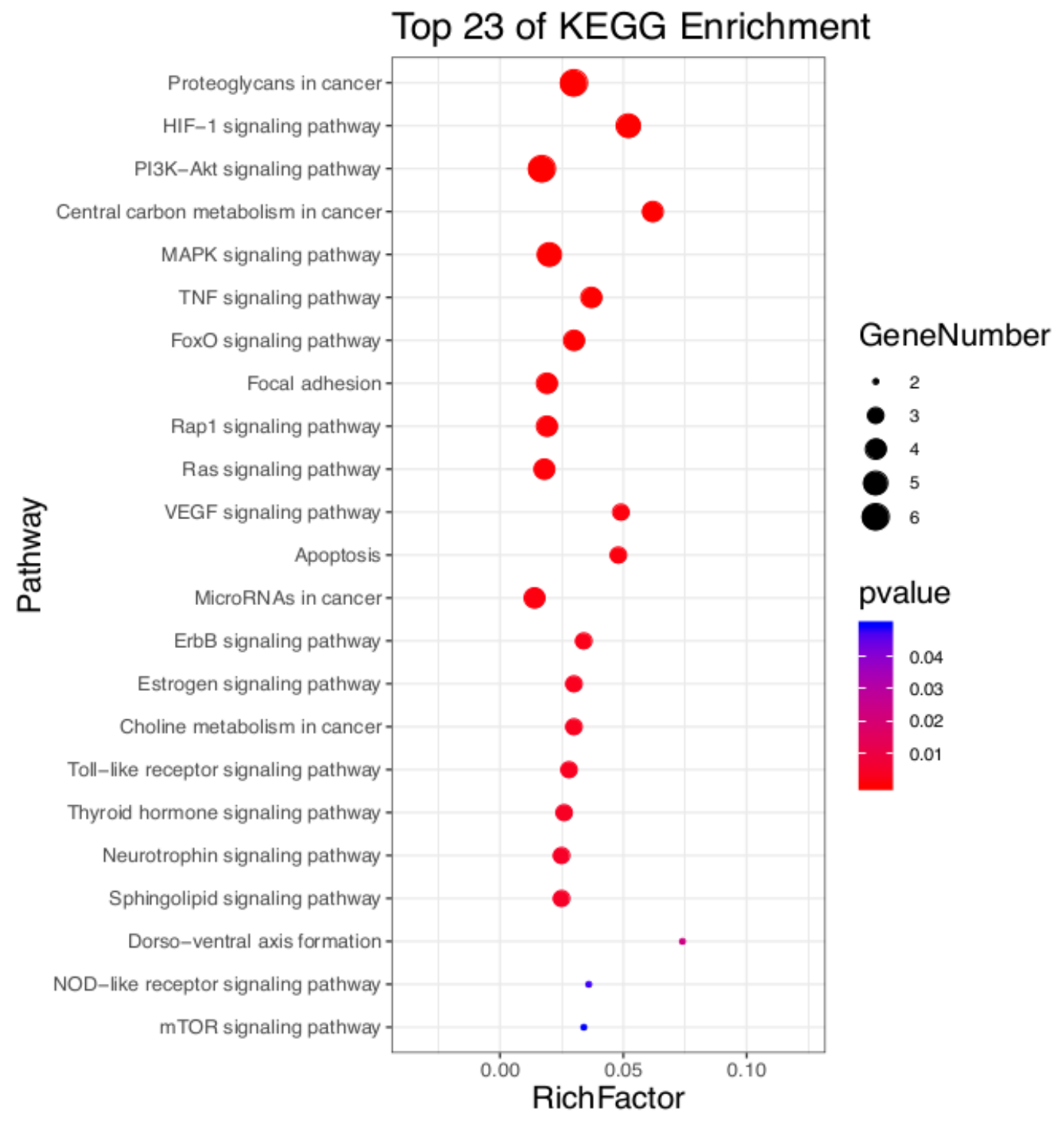

Figure 6

KEGG Pathway Enrichment. The size of the bubble represented the number of the gene hit on the pathway, the color of the bubble related to the Pvalue, the location of bubbles related to the RichFactor.

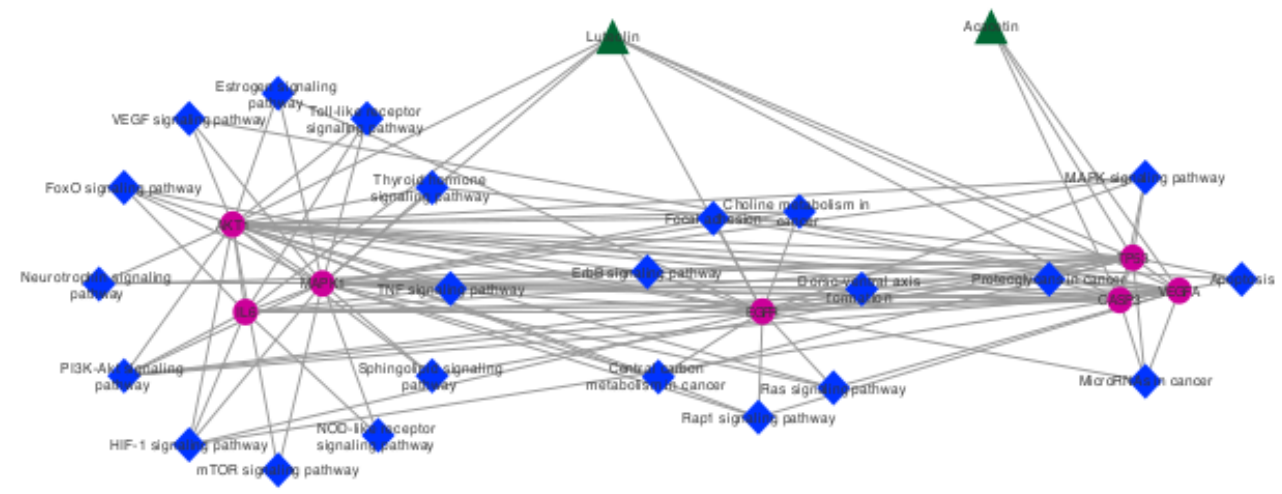

Figure 7

Compounds-targets-pathways network. Green triangle represented compounds, red circle represented targets, blue diamond represented pathways. 

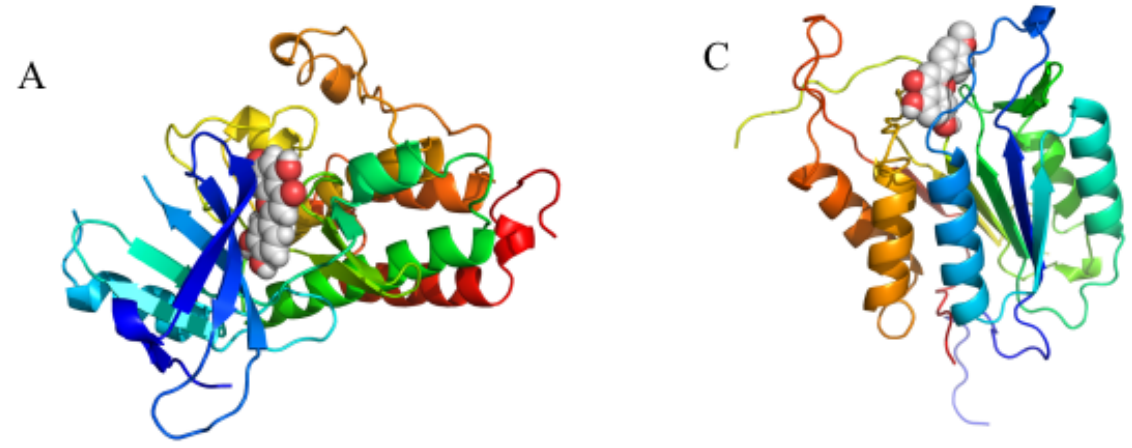

B

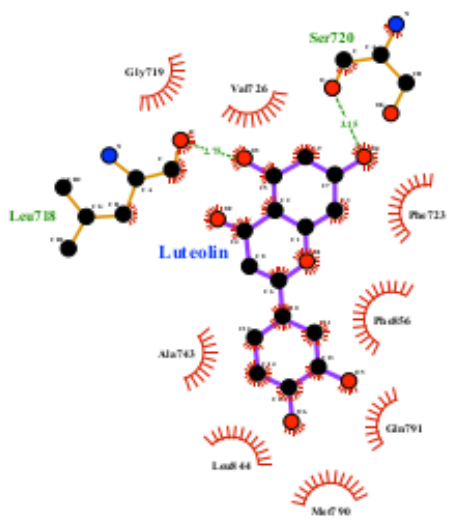

Luteolin_5UG9

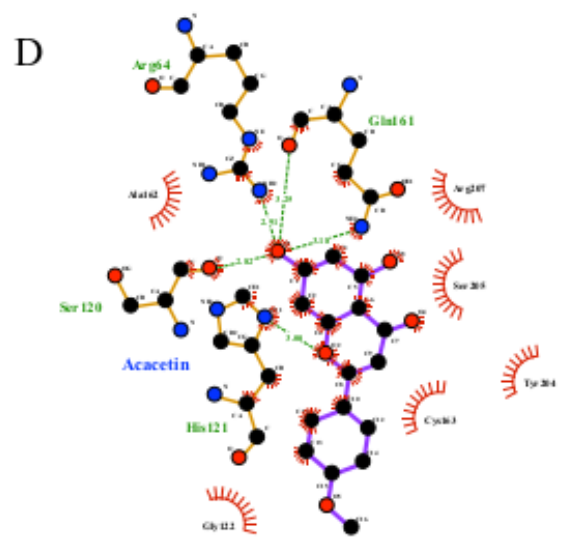

Acacetin_1NME

\section{Figure 8}

$\mathrm{A}$ and $\mathrm{C}$. The binding site was displayed by three-dimensional structure. The protein was displayed by ribbons, the compound was displayed by spheres. B and D. The interaction between compounds and protein residues was showed by two-dimensional structure.

\section{Supplementary Files}

This is a list of supplementary files associated with this preprint. Click to download.

- Table1.docx 